\title{
Ultra-thin Glass Film Coated with Graphene: A New Material for Spontaneous Emission Enhancement of Quantum Emitter
}

\author{
Lu Sun $\cdot$ Chun Jiang
}

Received: 8 December 2014/ Accepted: 5 February 2015/Published online: 4 April 2015

(C) The Author(s) 2015. This article is published with open access at Springerlink.com

\begin{abstract}
We propose an ultra-thin glass film coated with graphene as a new kind of surrounding material which can greatly enhance spontaneous emission rate (SER) of dipole emitter embedded in it. With properly designed parameters, numerical results show that SER-enhanced factors as high as $1.286 \times 10^{6}$ can be achieved. The influences of glass film thickness and chemical potential/doping level of graphene on spontaneous emission enhancement are also studied in this paper. A comparison is made between graphene and other coating materials such as gold and silver to see their performances in SER enhancement.
\end{abstract}

Keywords Graphene surface plasmons · Graphene plasmonics · Quantum electrodynamics · Superradiance · Subwavelength structures

\section{Introduction}

Since the discovery of Purcell effect in 1946, a lot of research interests have been focused on the radiation environment of an emitter [1]. Apart from manipulating the emitter, spontaneous emission rate (SER) can also be enhanced or suppressed by its surrounding materials, including metamaterials such as photonic crystals and metalinsulator-metal plasmonic structures [2-5]. Owing to the small mode volume, the SER-enhanced factors obtained in metamaterials are usually several orders higher than those in natural materials. However, losses are remarkable in these small mode volumes, e.g., energy will leak into free space from planar photonic crystals and metal resistance will cause dissipations in plasmonic structures. So the SER-enhanced factors are generally limited to the order of $10^{2}-10^{3}[2-5]$.

L. Sun · C. Jiang $(\bowtie)$

State Key Laboratory of Advanced Optical Communication

System and Networks, Shanghai Jiao Tong University,

Shanghai 200240, People's Republic of China

e-mail: cjiang@sjtu.edu.cn
Graphene, a monolayer of carbon atoms arranged in a two-dimensional hexagonal lattice, has attracted great research interests since it was first isolated from bulk graphite in 2004 [6]. The surface plasmon (SP) of doped graphene has lower losses compared to that of traditional plasmonic materials [7]. Its electromagnetic fields are highly confined to the graphene monolayer [8]. Therefore, graphene has become a promising material for SER enhancement.

Due to all the advantages mentioned above, graphene materials such as single-layer sheet, nanoribbon, and nanodisk have already been investigated for strong light-matter interaction [8-11]. However, since graphene is an atomically thick sheet of carbon, quantum emitter cannot be embedded into it and the electromagnetic fields radiated by the emitter are poorly confined. Thus, we consider constructing a new type of metamaterial made of graphene to improve it. In our previously reported work, we designed a double-layer graphene waveguide which greatly enhances the spontaneous emission of quantum emitter placed in it [12]. However, it requires static gate voltages to control the chemical potential of graphene, which makes it an active device. The electrodes attached to the graphene layers would bring great complexity to the fabrication processes. 
In this paper, we propose a new kind of metamaterial consisting of an ultra-thin glass film coated with doped graphene monolayers. The chemical potential of graphene is manipulated through chemical doping instead of electrical tuning, so it is more like a passive material than an active device. Since mid-infrared (MIR) light sources are essential for applications that include free-space communication, chemical and biomolecular sensing, and infrared spectroscopy [13, 14], we focus on the wavelength range from 3000 to $6000 \mathrm{~nm}$. With proper doping level, interband transitions are forbidden in graphene for this regime and SPs sustain lower losses [7]. Quantum emitters embedded in the glass will experience strong radiation reaction due to the graphene coating. Using finite-difference time-domain (FDTD) techniques, we prove that SER of quantum emitter has been greatly increased in the proposed material. Our design can make single-photon MIR source far more efficient in radiation, which could find applications in single-plasmon devices and quantum plasmonics [8]. Furthermore, stimulated emission is related to spontaneous emission by the boson nature of photon [15]. Therefore, it will be enhanced either due to the Purcell effect, and the radiation of conventional MIR light sources will also be improved by our material.

\section{Theoretical Analysis on the Proposed Metamaterial}

Quantum emitter, generally small in size, can be approximated by an electric dipole in physical models. The SER-enhanced factor or Purcell factor can be calculated by [16]:

$\frac{\gamma}{\gamma_{0}}=\frac{\mathbf{n}_{\mathbf{p}} \cdot \operatorname{Im}\left[\mathbf{G}\left(\mathbf{r}_{0}, \mathbf{r}_{0}, \omega\right)\right] \cdot \mathbf{n}_{\mathbf{p}}}{\mathbf{n}_{\mathbf{p}} \cdot \operatorname{Im}\left[\mathbf{G}_{0}\left(\mathbf{r}_{0}, \mathbf{r}_{0}, \omega\right)\right] \cdot \mathbf{n}_{\mathbf{p}}}$

where $\gamma$ is the SER of electric dipole in surrounding material, $\gamma_{0}$ is the one in free space, $\mathbf{n}_{\mathbf{p}}$ is the unit orientation vector of dipole moment $\mathbf{p}, \mathbf{G}\left(\mathbf{r}_{0}, \mathbf{r}_{0}, \omega\right)$ is the dyadic Green function in inhomogeneous environment, $\mathbf{G}_{0}\left(\mathbf{r}_{0}, \mathbf{r}_{0}\right.$, $\omega)$ is the dyadic Green function in free space, $\mathbf{r}_{0}$ and $\omega$ are the location and oscillation frequency of an emitter. The imaginary part of $\mathbf{G}(\mathbf{r}, \mathbf{r}, \omega)$ is related to the quantized electromagnetic field by [16]:

$\operatorname{Im}[\mathbf{G}(\mathbf{r}, \mathbf{r}, \omega)]=\frac{\pi c^{2}}{2 \omega} \sum_{\mathbf{k}} \mathbf{u}_{\mathbf{k}}^{*}\left(\mathbf{r}, \omega_{\mathbf{k}}\right) \mathbf{u}_{\mathbf{k}}\left(\mathbf{r}, \omega_{\mathbf{k}}\right) \delta\left(\omega-\omega_{\mathbf{k}}\right)$

where $\mathbf{u}_{\mathbf{k}}$ are the normal modes satisfying.

$$
\int_{V} \mathbf{u}_{\mathbf{k}}\left(\mathbf{r}, \omega_{\mathbf{k}}\right) \cdot \mathbf{u}_{\mathbf{k}^{\prime}}^{*}\left(\mathbf{r}, \omega_{\mathbf{k}^{\prime}}\right) d^{3} \mathbf{r}=\delta_{\mathbf{k k}^{\prime}}
$$

The integration runs over the entire mode volume $V$. If the field is tightly confined, i.e., it has a small mode volume, the modulus of $\mathbf{u}_{\mathbf{k}}$ must be large enough to ensure the unity of inner product of $\mathbf{u}_{\mathbf{k}}$ and $\mathbf{u}^{*} \mathbf{k}$. Then $\operatorname{Im}[\mathbf{G}(\mathbf{r}, \mathbf{r}$, $\omega)$ ] becomes considerable according to Eq. (2) and so is the Purcell factor according to Eq. (1). Thus, strong confinement of radiated fields is vital to SER enhancement, which inspires us to design metamaterials consisting of graphene.

We propose a new kind of metamaterial whose structure is illustrated in Fig. 1. The thickness of the glass film $d$ is usually on the order of tens of nanometers. Both the upper and lower facets of the film are covered with doped graphene monolayers. Glass is chosen as the middle layer for three reasons: (1) glass is transparent for MIR light and has low intrinsic losses in this regime; (2) graphene can be transferred to both sides of the glass layer by standard poly(methyl methacrylate) (PMMA) method [17]; (3) it also works as a supporting layer due to the ideal mechanical properties of glass. The size of structure in $x$ and $y$ direction is considerably large compared to the film thickness $d$ (the ratio of film thickness to graphene monolayer length in the $x$ direction is exaggerated in Fig. 1). The dipole emitter is placed at the center of the glass and its radiated fields are well confined by the extended graphene monolayers. The configuration of the proposed metamaterial is quite simple, which means its fabrication process is much easier than that of double-layer graphene waveguide reported in Ref. [12].

For MIR wavelengths considered here, the conductivity of graphene is dominated by intraband transition and can be simplified to a Drude-like expression as [18, 19]:

$\sigma_{\mathrm{g}}=\frac{i \mu_{\mathrm{c}} e^{2}}{\pi \hbar^{2}\left(\omega+i \tau^{-1}\right)}$

where $e$ is the charge of an electron, $\hbar$ is the reduced Planck's constant, $\omega$ is radian frequency, and $\tau$ is electron relaxation time. The chemical potential $\mu_{\mathrm{c}}$ can be tuned by
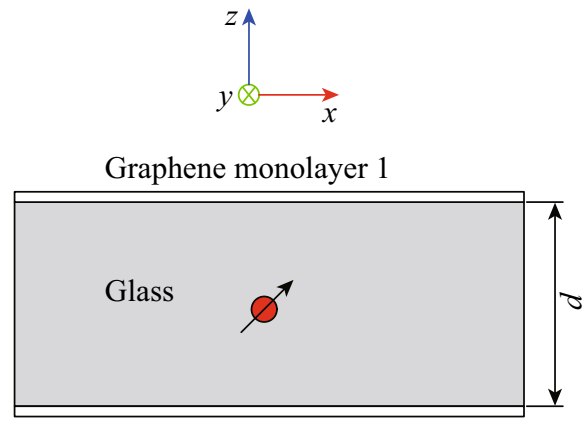

Graphene monolayer 2

Fig. 1 Schematic diagram of an ultra-thin glass film coated with graphene monolayer 1 and monolayer 2. Quantum emitter which is approximated by an electric dipole is placed at the center of glass film whose thickness is denoted as $d$ 
varying chemical doping levels during the fabrication, i.e., $\mu_{\mathrm{c}}=\hbar v_{\mathrm{f}} \sqrt{\pi n_{g}}$, where $n_{\mathrm{g}}$ is the carrier concentration of graphene and $v_{\mathrm{f}} \approx 10^{6} \mathrm{~m} / \mathrm{s}$ is the Fermi velocity in graphene. The mobility of graphene $\mu$ is set to $10,000 \mathrm{~cm}^{2} /$ $(\mathrm{V} s)$ which is a moderate value obtained in experiments [20]. It is related to electron relaxation time by $\tau=$ $\mu \mu_{\mathrm{c}} / e v_{\mathrm{f}}^{2}$. According to Eq. (4), the conductivity of graphene is calculated and plotted in Fig. 2a and b. The real part of conductivity is almost independent of chemical potential and increases monotonically with increasing wavelength while the imaginary part increases monotonically with increasing chemical potential and/or wavelength. Since $\operatorname{Im}\left(\sigma_{\mathrm{g}}\right)>0$, only transverse-magnetic (TM) SPs are supported by graphene [21,22]. They are supposed to be the main decay channels for quantum emitter discussed here.

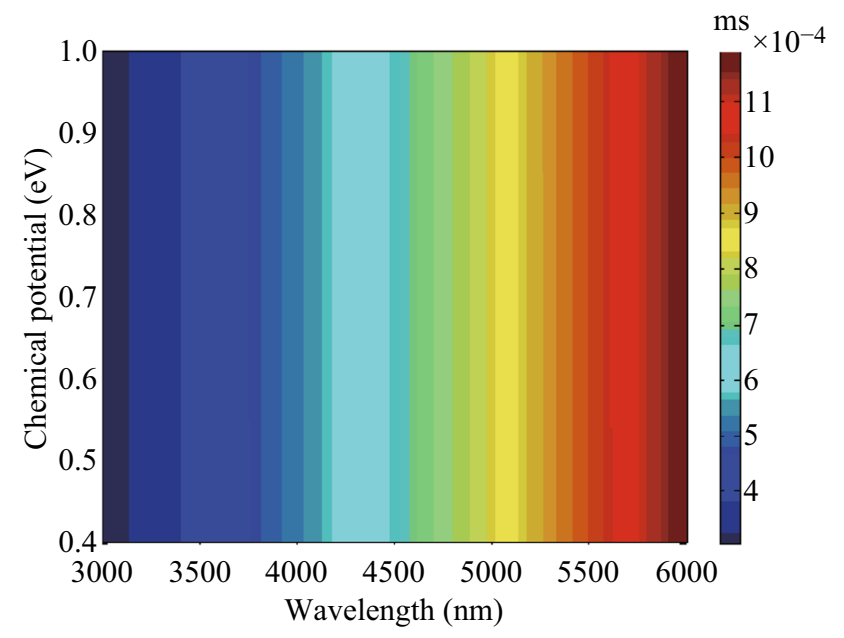

(a)

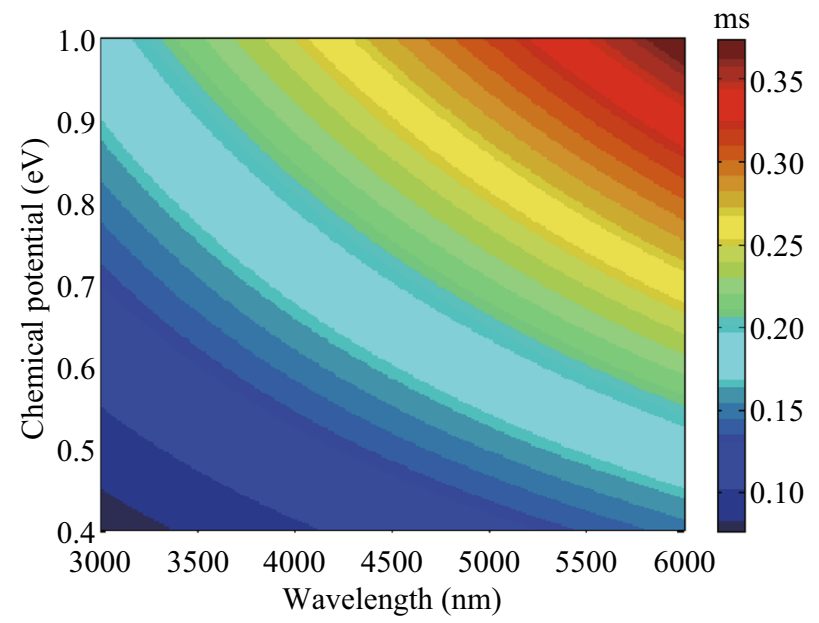

(b)

Fig. 2 a Real part and $\mathbf{b}$ imaginary part of the conductivity of graphene for various chemical potentials $(0.4-1 \mathrm{eV})$ and wavelengths $(3000-6000 \mathrm{~nm})$ in MIR regime

\section{Numerical Results and Discussion}

To further investigate the nature of SER enhancement in the new material, we use FDTD techniques to calculate the electromagnetic field distribution in the proposed structure. The related parameters are set as follows: glass film thickness $d=20 \mathrm{~nm}$, dipole moment oriented in the $z$ direction, chemical potential of graphene $\mu_{\mathrm{c}}=0.64 \mathrm{eV} . H_{x}$ components in graphene monolayer 1 and monolayer 2 have the same field pattern (Fig. 3a). As we can see, the energy of electric dipole is efficiently coupled into TM SPs of graphene, which is one of the most important decay channels in the near field. The field pattern of $H_{x}$ component in the $Y O Z$ plane is shown in Fig. 3b. It is obvious that electromagnetic fields are confined between two graphene monolayers, i.e., inside the glass film. The SER-enhanced factors can be calculated by $\gamma / \gamma_{0}=P / P_{0}$, where $P$ is the power radiated by electric dipole in surrounding material and $P_{0}$ is the one in free space. We conduct all the numerical calculations using commercial software called Lumerical FDTD solution. The total power radiated by the dipole is calculated by measuring the power flow out of a small volume which contains the source. In our simulations, we set six power monitors on the faces of a box surrounding the dipole. We find the highest SER-enhanced factor peak in the MIR regime and compare it with those of single-layer graphene sheet and nanoribbon. The results are shown in Fig. 3c. Figure 3d and e shows the schematics of single-layer graphene sheet and nanoribbon and the corresponding $H_{x}$ field distributions in them. The nanoribbon width is set to $100 \mathrm{~nm}$. Dipole emitters are all placed $10 \mathrm{~nm}$ away from graphene monolayers. The chemical potentials of the single-layer graphene sheet and nanoribbon are both $\mu_{\mathrm{c}}=0.64 \mathrm{eV}$ which corresponds to a doping density of $3.0 \times 10^{17} \mathrm{~m}^{-3}$. The SER-enhanced factor of ultra-thin glass film coated with graphene reaches its maximum of $1.286 \times 10^{6}$ at $5243 \mathrm{~nm}$, which is about three times and 12 times larger than that of single-layer nanoribbon and single-layer sheet, respectively. We can also see that the magnitude of $H_{x}$ field in ultra-thin glass film coated with graphene is much stronger than those in single-layer graphene sheet and nanoribbon. These results confirm the theories given in Sect. 2 that confinement of radiated fields is of great importance for SER enhancement, considering that ultra-thin glass film coated with graphene has better radiation field confinement than single-layer graphene sheet and nanoribbon.

Next we investigate the influence of glass film thickness on SER-enhanced factor. The film thickness varies from 20 to $50 \mathrm{~nm}$ with a step of $10 \mathrm{~nm}$. Other parameters are kept the same as in Fig. 3. The maximum SER-enhanced factor decreases monotonically with increasing film thickness (Fig. 4). This phenomenon can be explained as follows: 


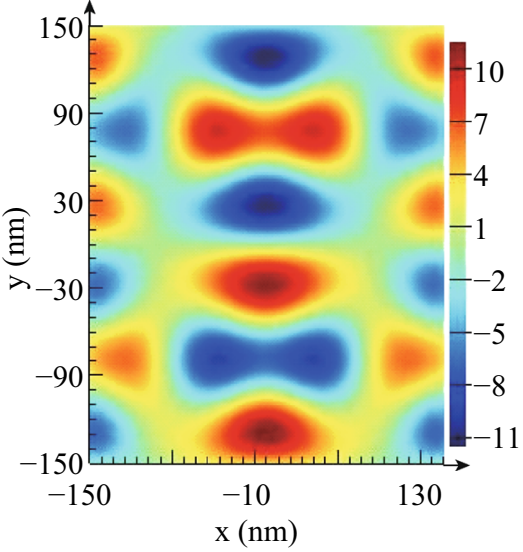

(a)

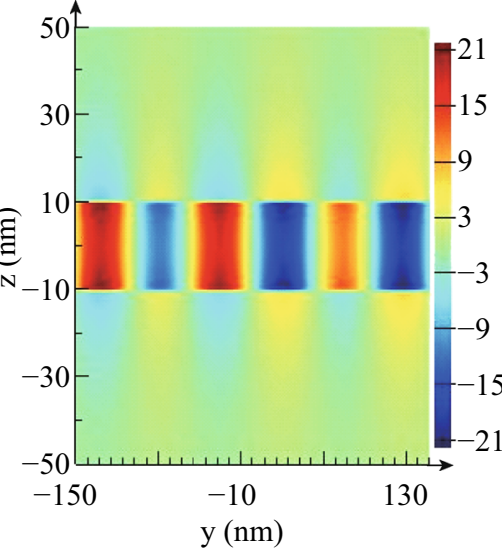

(b)

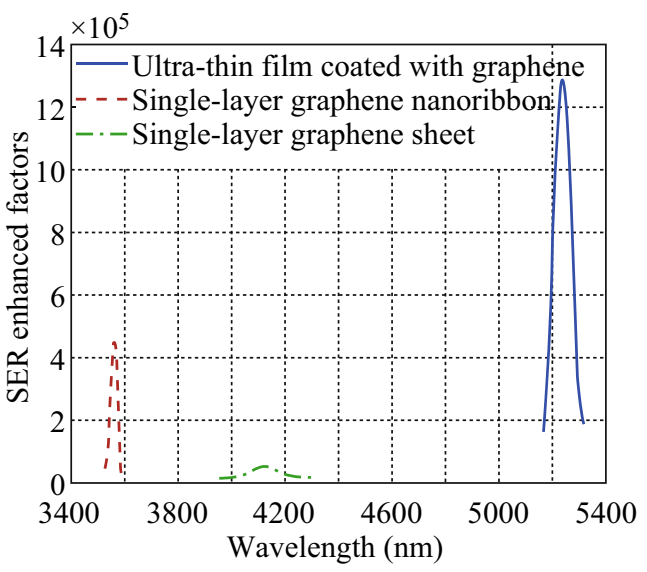

(c)
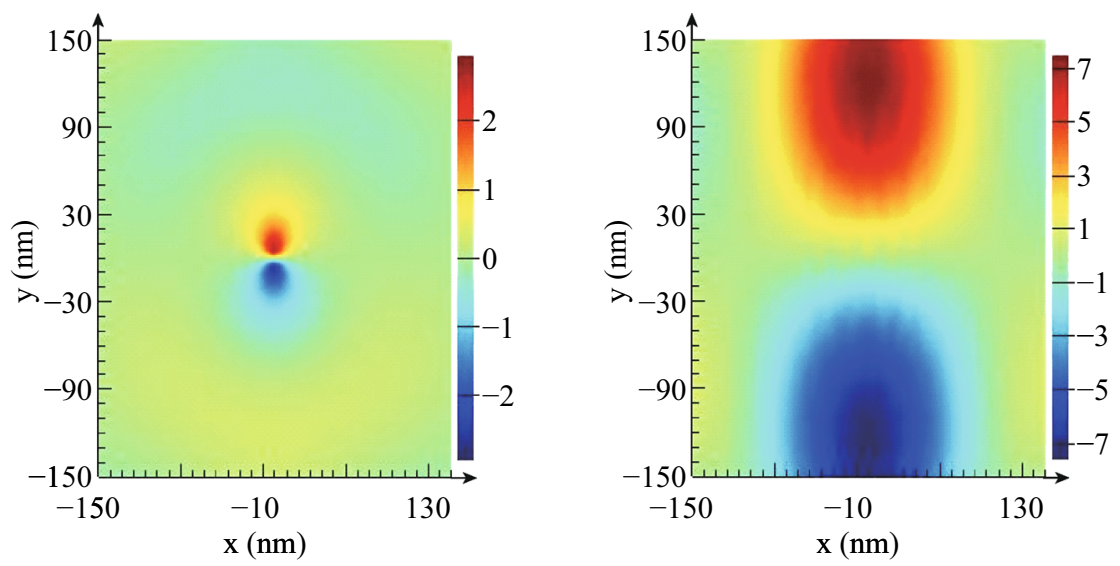

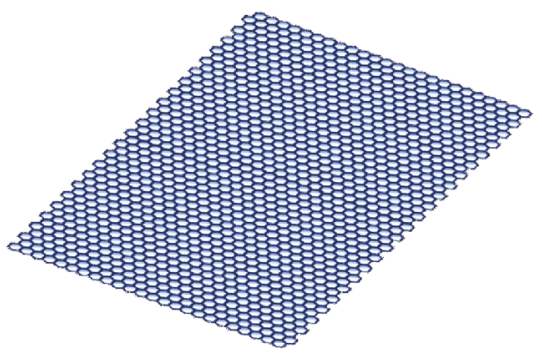

(d)

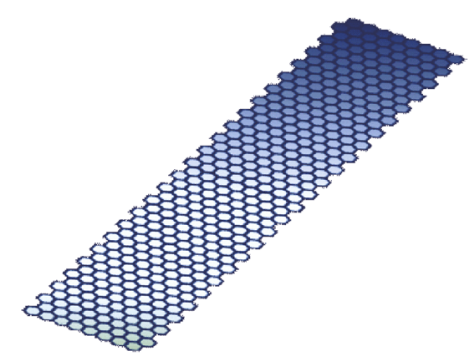

(e)

Fig. 3 a $H_{x}$ component in a graphene monolayer and $\mathbf{b}$ the $Y O Z$ plane of ultra-thin glass film coated with graphene. The film thickness $d=20 \mathrm{~nm}$, dipole moment is orientated in the $z$ direction, and chemical potential of graphene $\mu_{\mathrm{c}}=0.64 \mathrm{eV}$. c SER-enhanced factors of electric dipole near ultra-thin glass film coated with graphene (blue solid line), single-layer graphene sheet (green dash-dotted line), and single-layer graphene nanoribbon (red dashed line) of $100 \mathrm{~nm}$ in width. Schematics and $H_{x}$ field distributions of single-layer graphene d sheet and e nanoribbon. Dipoles are placed $10 \mathrm{~nm}$ away from graphene monolayers and the chemical potentials of graphene are $\mu_{\mathrm{c}}=0.64 \mathrm{eV}$. (Color figure online)

with larger separation distance between graphene double layers, the radiated fields are less confined, which leads to weaker light-matter interactions. For film thickness less than $20 \mathrm{~nm}$, the SER enhancement will be stronger. However, the complexity of fabrication will increase enormously. So we do not discuss thinner film thicknesses in this paper.
Another parameter which will affect the SER-enhanced factor is the doping level or chemical potential of graphene. The chemical potentials of $0.4,0.64$, and $1 \mathrm{eV}$ which correspond respectively to doping densities of $1.17 \times 10^{17}$, $3 \times 10^{17}$, and $7.32 \times 10^{17} \mathrm{~m}^{-3}$ are explored in this paper. Chemical potential lower than $0.4 \mathrm{eV}$ is unconcerned in our discussion because in that case interband transition 


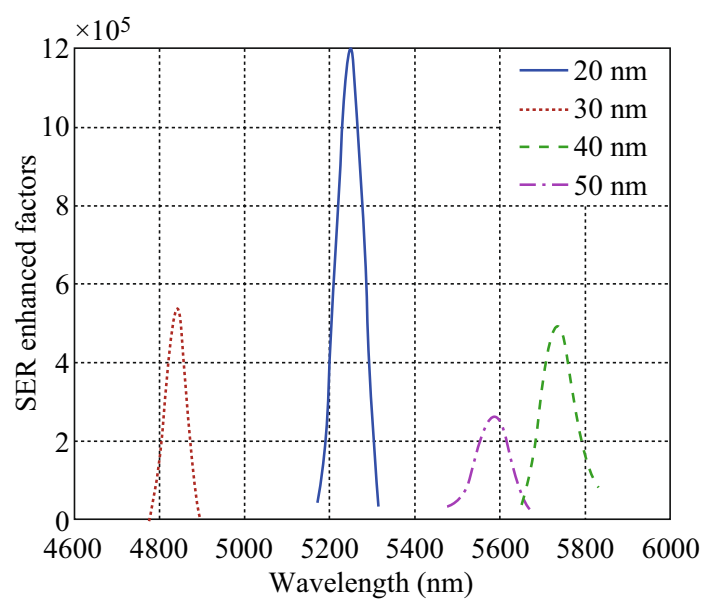

Fig. 4 SER-enhanced factors for glass film thickness of 20 (blue solid line), 30 (red dashed line), 40 (green dotted line), and $50 \mathrm{~nm}$ (purple dash-dotted line). (Color figure online)

occurs in graphene with incident photons of MIR wavelengths and brings high losses to graphene SPs. The SERenhanced factors are numerically calculated using FDTD techniques and the results are shown in Fig. 5. It is obvious that the metamaterial made of graphene with a higher chemical potential or doping level acquires a larger SERenhanced factor. It can be explained considering the nature of graphene TM SPs whose propagation constant $q$ and outof-plane decay coefficients $Q_{1}, Q_{2}$ can be expressed as [7]:

$q \approx Q_{1} \approx Q_{2} \approx \varepsilon_{0}\left(\varepsilon_{\mathrm{r} 1}+\varepsilon_{\mathrm{r} 2}\right) \frac{i \omega}{\sigma(\omega)}$

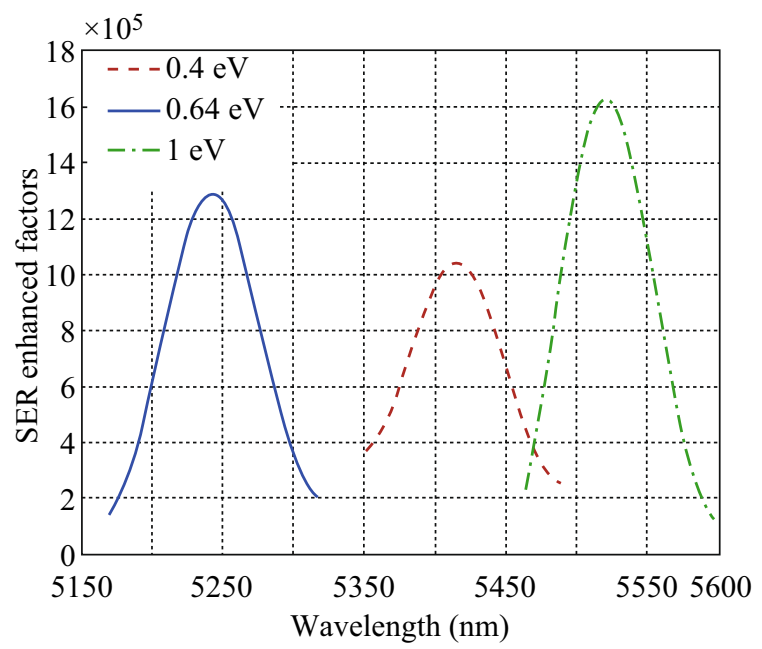

Fig. 5 SER-enhanced factors for chemical potential of 0.4 (red dashed line), 0.64 (blue solid line), and $1.0 \mathrm{eV}$ (green dash-dotted line). (Color figure online) for $q \gg \omega / c \varepsilon_{1}$ and $\varepsilon_{2}$ are the dielectric constants of surrounding medium 1 and medium 2, respectively. $Q_{1}$ and $Q_{2}$ are the corresponding decay coefficients. Since the imaginary part of the conductivity of graphene is several orders larger than its real part, only the imaginary part needs to be considered in Eq. (5). The three resonance wavelengths in Fig. 5 are close to each other in the spectrum, so the imaginary part of conductivity mainly depends on the chemical potential of graphene (see Fig. 2). They are $0.1466,0.2097$, and $0.3450 \mathrm{mS}$ which correspond to decay coefficients of $5.15 \times 10^{7}, 3.72 \times 10^{7}$, and $2.15 \times 10^{7}$ $\mathrm{m}^{-1}$ for $0.4,0.64$, and $1 \mathrm{eV}$, respectively. The fields of graphene TM SPs decay exponentially along the $y$ direction. A higher decay coefficient will lead to a weaker interaction between the graphene and electric dipole placed $10 \mathrm{~nm}$ away from it. Thus, we can conclude that the dipole energy is coupled more efficiently to graphene with a higher chemical potential, which results in a larger SERenhanced factor. This fact explains the phenomenon observed in Fig. 5.

The proposed metamaterial also enjoys high flexibility in several ways. For example, the frequencies at which SERs are enhanced can be selected by either tuning the chemical potential of graphene $\mu_{\mathrm{c}}$ or changing the glass film thickness $d$, as indicated in Figs. 4 and 5. As for applications, the metamaterial can be directly integrated with quantum emitters as the surrounding medium to form highly efficient subwavelength single-photon sources.

However, there exist some factors that limit the SERenhanced factor. Chemical doping will inevitably introduce scattering source and thus degrade the mobility of intrinsic graphene [23]. Meanwhile, supporting substrate will also bring additional scattering mechanisms such as interface impurity scattering and remote phonon scattering [24]. These cause the degradation of graphene mobility as well. For lower mobility, e.g., $\mu=5000 \mathrm{~cm}^{2} /(\mathrm{V} \mathrm{s})$, an SERenhanced factor of $1.197 \times 10^{6}$ can be achieved in the structure. So in reality, the SER-enhanced factor will be lower than that obtained without taking mobility degradation into consideration, but still on the order of $10^{6}$.

Finally, we compare graphene with other coating materials such as gold and silver. Two graphene monolayers shown in Fig. 1 are now replaced by $20 \mathrm{~nm}$-thick gold/ silver films. The maximum SER-enhanced factors of gold and silver coatings are given in Fig. 6a. The SER-enhanced factor of silver coating is a little higher than that of gold one due to its lower intrinsic losses at optical frequencies. Although SER-enhanced factors of over 500 can be achieved by both coating materials, they are still several orders lower than that of graphene. The reasons for the extremely high SER-enhanced factor of graphene-based metamaterial can be figured out after studying the photonic 


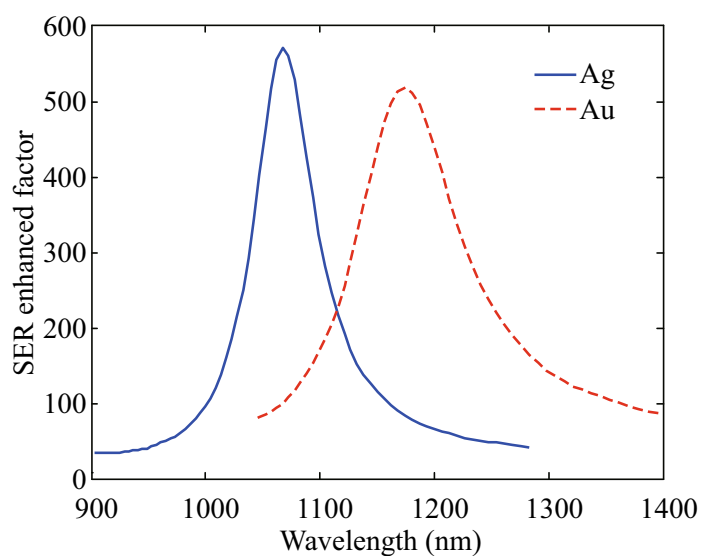

(a)
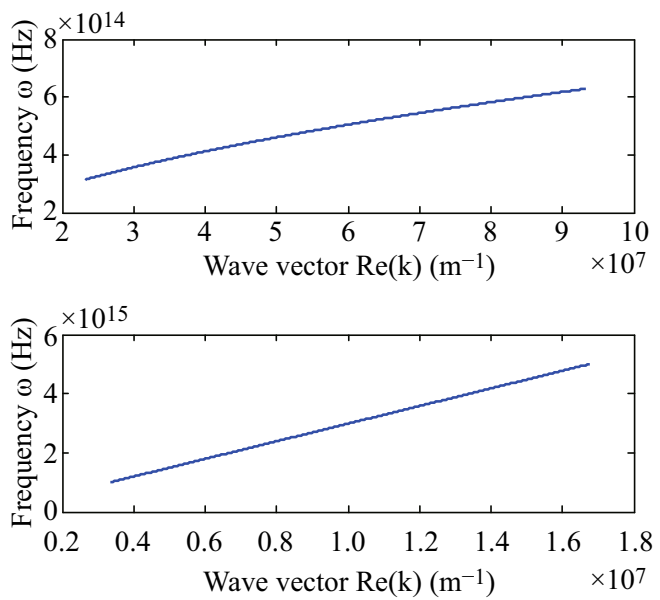

(b)

Fig. 6 a SER-enhanced factors for gold (red dashed line) and silver (blue solid line) coatings, b dispersion relationships of TM SPs on silica-graphene and silica-silver interfaces. (Color figure online)

density of states of graphene TM SPs. The plasmon density of states $\rho(\hbar \omega)$ can be written as [25].

$\rho(\hbar \omega)=\frac{L^{2}}{4 \pi} \frac{\mathrm{d}\left(k^{2}\right)}{\mathrm{d}(\hbar \omega)}$

where $L^{2}$ is the in-plane quantization area, $k$ is the in-plane wave vector of plasmon. The dispersion relationships of TM SPs on silica-silver and silica-graphene interfaces are plotted in Fig. 6b according to which the photonic density of states of graphene SPs is generally three orders larger than that of silver ones, i.e., $\rho_{\text {graphene }} / \rho_{\text {silver }} \sim 10^{3}$. It is in high agreement with the ratio between SER-enhanced factors of glass films covered by graphene and silver coatings, which can be predicted by the Fermi's golden rule. Therefore, we have come to the conclusion that the dispersion relationship of graphene TM SPs contributes tremendously to plasmon density of states in the space, which leads to a super high SER-enhanced factor of ultrathin glass film coated with graphene.

\section{Conclusion}

In conclusion, we proposed an ultra-thin glass film coated with graphene where the SERs of embedded quantum emitter were multiplied by a factor typically on the order of $10^{6}$. The influences of glass film thickness and chemical potential/doping level of graphene on SER-enhanced factor were also studied. A comparison of graphene and other coating materials including gold and silver was made in terms of SER enhancement. Explanations and quantitative analysis were given to the observed phenomena. Different from previously reported works, the metamaterial we proposed benefits from the nature of graphene SPs. The radiated fields are better confined in the ultra-thin glass film coated with graphene, which gives rise to stronger light-matter interactions and higher SER-enhanced factors. It has great potential to work as the surrounding material of highly efficient quantum emitters for novel single-plasmon devices and quantum plasmonic applications in the near future.

Acknowledgments This work was supported in part by the National Natural Science Foundation of China (Grant No. 61177056) and the Cultivation Fund of the Key Scientific and Technical Innovation Project, Ministry of Education of China (Grant No. 708038).

Open Access This article is distributed under the terms of the Creative Commons Attribution 4.0 International License (http:// creativecommons.org/licenses/by/4.0/), which permits unrestricted use, distribution, and reproduction in any medium, provided you give appropriate credit to the original author(s) and the source, provide a link to the Creative Commons license, and indicate if changes were made.

\section{References}

1. E.M. Purcell, Spontaneous emission probabilities at radio frequencies. Phys. Rev. 69, 681 (1946). doi:10.1103/PhysRev.69. 674.2

2. V.S.C.M. Rao, S. Hughes, Numerical study of exact Purcell factors in finite-size planar photonic crystal waveguides. Opt. Lett. 33(14), 1587-1589 (2008). doi:10.1364/OL.33.001587

3. M. Kuttge, F.J.G. de Abajo, A. Polman, Ultrasmall mode volume plasmonic nanodisk resonators. Nano Lett. 10(5), 1537-1541 (2010). doi:10.1021/n1902546r

4. P. Kristensen, C.V. Vlack, S. Hughes, Generalized effective mode volume for leaky optical cavities. Opt. Lett. 37(10), 1649-1651 (2012). doi:10.1364/OL.37.001649

5. H. Iwase, D. Enlund, J. Vučković, Analysis of the Purcell effect in photonic and plasmonic crystals with losses. Opt. Express 18(16), 16546-16560 (2010). doi:10.1364/OE.18.016546

6. A.K. Geim, K.S. Novoselov, The rise of graphene. Nat. Mater. 6 , 183-191 (2007). doi:10.1038/nmat1849

7. M. Jablan, H. Buljan, M. Soljačić, Plasmonics in graphene at infrared frequencies. Phys. Rev. B 80, 245435 (2009). doi:10. 1103/PhysRevB.80.245435

8. F.H.L. Koppens, D.E. Chang, F.J.G. de Abajo, Graphene plasmonics: a platform for strong light-matter interactions. Nano Lett. 11(8), 3370-3377 (2011). doi:10.1021/nl201771h 
9. G.W. Hanson, E. Forati, W. Linz, A.B. Yakovlev, Excitation of terahertz surface plasmons on graphene surfaces by an elementary dipole and quantum emitter: strong electrodynamic effect of dielectric support. Phys. Rev. B 86, 235440 (2012). doi:10.1103/ PhysRevB.86.235440

10. P.A. Huidobro, AYu. Nikitin, C. González-Ballestero, L. MartínMoreno, F.J. García-Vidal, Superradiance mediated by graphene surface plasmons. Phys. Rev. B 85, 155438 (2012). doi:10.1103/ PhysRevB.85.155438

11. AYu. Nikitin, F. Guinea, F.J. García-Vidal, L. Martín-Moreno, Fields radiated by a nanoemitter in a graphene sheet. Phys. Rev. B 84, 195446 (2011). doi:10.1103/PhysRevB.84.195446

12. L. Sun, B. Tang, C. Jiang, Enhanced spontaneous emission of mid-infrared dipole emitter in double-layer graphene waveguide. Opt. Express 22(22), 26487-26497 (2014). doi:10.1364/OE.22. 026487

13. R.A. Soref, S.J. Emelett, W.R. Buchwald, Silicon waveguided components for the long-wave infrared region. J. Opt. A 8 , 840-848 (2006). doi:10.1088/1464-4258/8/10/004

14. V. Raghunathan, D. Borlaug, R. Rice, B. Jalali, Demonstration of a mid-infrared silicon Raman amplifier. Opt. Express 15, 14355-14362 (2007). doi:10.1364/OE.15.014355

15. P. Meystre, M. Sargent, Elements of Quantum Optics, 4th edn. (Springer, New York, 2007), pp. 299-349. doi:10.1007/978-3662-03877-2

16. L. Novotny, B. Hecht, Principles of Nano-optics, 2nd edn. (Cambridge University Press, New York, 2012), pp. 250-303. doi:10.1017/CBO9780511794193

17. A. Reina, H. Son, L. Jiao, B. Fan, M.S. Dresselhaus, Z. Liu, J. Kong, Transferring and identification of single- and few-layer graphene on arbitrary substrates. J. Phys. Chem. C 112, 17741-17744 (2008). doi:10.1021/jp807380s

18. L.A. Falkovsky, Optical properties of graphene. J. Phys.: Conf. Ser. 129(1), 012004 (2008). doi:10.1088/1742-6596/129/1/ 012004

19. M.I. Katsnelson, Graphene: Carbon in Two Dimensions (Cambridge University Press, New York, 2012), pp. 161-184. doi:10. 1017/CBO9781139031080

20. L. Gao, J. Shu, C. Qiu, Q. Xu, Excitation of plasmonic waves in graphene by guided-mode resonances. ACS Nano 6(9), 7806-7813 (2012). doi:10.1021/nn301888e

21. A. Vakil, N. Engheta, Transformation optics using graphene. Science 332(6035), 1291-1294 (2011). doi:10.1126/science. 1202691

22. S.A. Mikhailov, K. Ziegler, New electromagnetic mode in graphene. Phys. Rev. Lett. 99, 016803 (2007). doi:10.1103/Phys RevLett.99.016803

23. D.B. Farmer, H.-Y. Chiu, Y.-M. Lin, K.A. Jenkins, F. Xia, P. Avouris, Utilization of a buffered dielectric to achieve high fieldeffect carrier mobility in graphene transistors. Nano Lett. 9(12), 4474-4478 (2009). doi:10.1021/n1902788u

24. T. Fang, A. Konar, H. Xing, D. Jena, Mobility in semiconducting graphene nanoribbons: phonon, impurity, and edge roughness scattering. Phys. Rev. B 78, 205403 (2008). doi:10.1103/Phys RevB.78.205403

25. I. Gontijo, M. Boroditsky, E. Yablonovitch, S. Keller, U.K. Mishra, S.P. DenBaars, Coupling of InGaN quantum-well photoluminescence to silver surface plasmons. Phys. Rev. B 60(16), 11564-11567 (1999). doi:10.1103/PhysRevB.60.11564 